\title{
Erratum
}

\section{Concise Asymmetric Total Synthesis of ent-Guadinomic Acid from an Epoxy Alkenol}

Hyemi Kim, Myung-Yeol Kim, Jinsung Tae* Synlett 2009, 2949.

The advance online publicaton (e-First) version of this article contained an error in Scheme 5.

This mistake has been corrected for both the current online version and the print publication. 